ISSN 1112-9867

Available online at

http://www.jfas.info

\title{
RETROSPECTIVE STUDY OF EPIDEMIOLOGICAL, CLINICOPATHOLOGICAL AND BIOLOGICAL PROFILS OF 62 COLORECTAL CANCERS CASES IN JIJEL PROVENCE (ALGERIA)
}

\author{
A. Abbes ${ }^{1}$, H. Rechreche ${ }^{1 *}$, R. Brinet ${ }^{1}$, S. Boulkhiout ${ }^{1}$, N. Souilah ${ }^{1}$, M. Benali ${ }^{2}$ \\ ${ }^{1}$ Laboratoire de Biologie Moléculaire et Cellulaire (MCBL), Département BMC, Université \\ Mohamed Sedik Benyahia, Jijel, Algérie \\ ${ }^{2}$ Laboratoire de Biotoxicologie, Département de Biologie, Université Djillali Liabes, \\ Sidi-Bel-Abbes, Algerie
}

Received: 03 February 2017 / Accepted: 12 October 2017 / Published online: 01 January 2018

\begin{abstract}
In Algeria, the CRC wing and become the first digestive cancer in both sexes, outperforming stomach cancer. To enrich the Algerian cancer registries, we analyzed the profiles of patients with these cancers in Jijel Willaya. This was a retrospective and descriptive analysis of epidemiological, clinicopathological and biological profiles of $62 \mathrm{CRC}$ cases. We found that the $\mathrm{CRC}$ represented the first type of digestive cancers in which the three quarters were colon cancers. The most affected age group was 60-70 years with a male predominance and an average age of 56.20 years. The bleeding and abdominal pain were the majority telltale signs. The combined chemotherapy has been standardized with all patients and the Lieberkühnien adenocarcinoma was the major histological form. The disease issue and the choice of therapy depended on the K-RAS gene mutations. Our results were often compatible with the available literature and may provide reliable and relevant data on this disease.
\end{abstract}

Key words: Colorectal cancer; Epidemiology; Therapy; Adenocarcinoma; K-RAS gene

Author Correspondence, E-mail : horechre@gmail.com

doi: http://dx.doi.org/10.4314/jfas.v10i1.5 


\section{INTRODUCTION}

Les cancers colorectaux (CCR) constituent un problème majeur de santé publique dans les pays développés en raison de leur fréquence et leur gravité. Annuellement, un total de près d'un million deux cent mille cas de cette pathologie est diagnostiqué et près d'un demi-million de personnes en meurent [1]. Les CCR occupent le troisième rang des cancers malins en termes d'incidence et de mortalité [2]. À l'heure actuelle, on ne connaît pas la cause de la formation et le développement des adénomes, on sait toutefois que le tabagisme, l'alimentation riche en graisses et pauvre en fibres alimentaires, la consommation excessive d'alcool et la surcharge pondérale sont des facteurs de risque [3].

En Algérie, l'incidence des CCR demeure faible par rapport aux pays développés, en dépit du fait qu'elle est en constante augmentation. En effet, les CCR sont devenus les premiers cancers digestifs chez les deux sexes, surclassant le cancer de l'estomac, et comptent parmi les cancers les plus fréquents, occupant ainsi la troisième place de l'ensemble des cancers [4]. De nombreuses études ont été dédiées aux CCR dans le monde et qui visaient l'amélioration des pratiques médicales, aussi bien sur le plan diagnostique que thérapeutique. Par ailleurs, la recherche épidémiologique représente un moyen de faire évoluer la situation, elle apporte des renseignements sur la fréquence de ce cancer, le terrain sur lequel il survient, les maladies susceptibles de transformation maligne et les facteurs de l'environnement favorisant leur survenue. En effet, ces données permettent d'envisager une stratégie nationale efficace de lutte contre les CCR. Cependant, il est très regrettable de constater qu'à ce jour les travaux de recherche qui ont été consacrés aux CCR en Algérie sont rares, limités et fragmentaires. De plus, la fréquence et l'incidence des CCR, ainsi que la mortalité par cette pathologie ne sont pas estimées avec précision, par manque de registre de cancer. Ceci s'explique par l'absence d'une politique de santé publique adéquate en vue de permettre un diagnostic précoce et une prise en charge correcte ce qui fait de ces cancers un tueur froid et redoutable [5].

C'est dans ce contexte que nous avons entrepris ce travail dont le but essentiel était de déterminer les profils épidémiologique, clinico-pathologique et biologique de 62 patients atteints de CCR et résidants dans la wilaya de Jijel, ce qui pourrait participer au développement de stratégies pour améliorer leur prise en charge. 


\section{EXPERIMENTAL}

\subsection{Population et type d'étude}

Il s'agissait d'une analyse rétrospective et descriptive des profils épidémiologique, clinico-pathologique et biologique de 62 cas de CCR, pris en charge par le Service d'Oncologie de l'Hôpital Mohamed Essedik Benyahia (MEB) pendant la période 2012-14. Elle a inclus tous les cas de cancers primitifs du côlon et/ou du rectum, d'âges compris entre 20 et 80 ans et résidant dans la Wilaya de Jijel. Le volet épidémiologique a consisté à la détermination de l'incidence des CCR et leur comparaison aux différents types de cancers. Les paramètres épidémiologiques étudiés comprennent le sexe, la tranche d'âges, la distribution géographique, les antécédents personnels et familiaux.

Le volet clinique et paraclinique a permis de préciser les circonstances de la découverte de la pathologie, l'évolution des symptômes et les examens paracliniques réalisés pour chaque patient. Ainsi, les patients ont bénéficié d'explorations fonctionnelles et radiologiques, de bilans biologiques (Transaminases, phosphatase alcaline ou PAL, globules rouges et blanc), ainsi que du dosage des marqueurs tumoraux (Antigène carcino-embryonnaire ou ACE et antigène carbohydrate 19-9 ou CA19-9). L'examen anatomopathologique permet de déterminer le type histologique, le degré de différenciation, l'architecture et la stadification des CCR. Afin d'apprécier l'état basal des tissus, nous avons analysé par microscopie optique les tissus cancéreux de deux patients, après coloration par l'hématoxyline/éosine. En outre, nous avons tenté d'établir des corrélations potentielles entre les mutations du gène K-RAS et certains paramètres tels que le sexe, le siège de la tumeur et le stade de différenciation.

Enfin, nous avons analysé les données des patients qui ont bénéficié d'une chirurgie curative ou palliative, d'une radiothérapie ou d'une chimiothérapie seule ou combinée.

\subsection{Recueil, saisie et analyse des données}

Le recueil des données des différents paramètres étudiés a été réalisé en élaborant une fiche de renseignement qui comporte les informations enregistrées à partir des dossiers de malades. Leur saisie a été effectuée ultérieurement par le logiciel Epi-info7 qui a permis de créer des questionnaires, de saisir les réponses à ces questionnaires et d'appliquer des statistiques. Ce logiciel est créé par le Centre de Surveillance des Maladies d'Atlanta aux États-Unis (CSM), 
en collaboration avec l'Organisation Mondiale de la Santé (OMS). Afin de traiter les données recueillies, nous avons suivi deux types de statistiques : descriptives (Tableaux et histogrammes) et analytiques (écart type et p-value).

\section{RESULTS AND DISCUSSION}

\subsection{Profils épidémiologiques}

Dans un premier temps, nous avons cherché à déterminer l'incidence des CCR dans notre Wilaya. Ainsi, nous avons colligé un total de 474 cas de cancers recensés au niveau du Service d'Oncologie de l'Hôpital MEB, où les femmes et les hommes ont respectivement représentés 330 et 144 cas. Nous avons observé que les CCR se plaçaient en $2^{\text {ème }}$ position de l'ensemble des cancers étudiés (13\%), précédés par les cancers du sein (41\%) et suivis par les cancers de l'appareil respiratoire (6\%) (Figure1). Nos résultats ont été divergents avec ceux rapportés par de nombreux travaux qui ont démontré que les CCR étaient les $3^{\text {èmes }}$ cancers, les plus fréquemment diagnostiqués chez les hommes, après les cancers du poumon et de la prostate, et les $2^{\text {èmes }}$ chez les femmes après le cancer du sein [6]. De plus, nous avons constaté que l'incidence des CCR représentait $52 \%$ des cancers digestifs, ce qui est tout à fait concordant avec des études rapportées par El Housse et coll., selon lesquelles, les CCR occupaient la première place des cancers digestifs [7].

Dans un deuxième temps, nous avons évalué la répartition des CCR en fonction du sexe et de la tranche d'âges. Nous avons constaté que 53\% des sujets atteints de CCR étaient de sexe masculin (Figure 02). Ces résultats sont similaires aux données obtenues par une étude épidémiologique sur les CCR à l'Ouest algérien, selon laquelle, une prédominance masculine a été constatée, soit $54 \%$ chez les hommes contre $46 \%$ chez les femmes [8]. Le pic de fréquence et la moyenne d'âges des CCR pour les deux sexes réunis ont été évalués à 60-70 ans et 56,20 ans, respectivement. La relation âge/sexe n'était pas significative $(p=0.45)$. Ces résultats concordent avec une étude marocaine réalisée par Sentissi et coll. qui ont proposé un âge moyen de 54 ans pour les deux sexes [9]. Cependant, cette valeur est inférieure d'une dizaine d'années par rapport à l'âge moyen des populations vieillissantes des pays développés. Les patients âgés de plus que 50 ans ont représenté $69 \%$ de l'ensemble des cas, contre 31\% 
des patients de moins de 50 ans (Figure 2). De tels résultats sont similaires aux données publiées par l'Institut National du Cancer de France en 2012 et qui renforcent l'idée selon laquelle un âge supérieur à 50 ans constitue un facteur de risque majeur de développer un CCR.

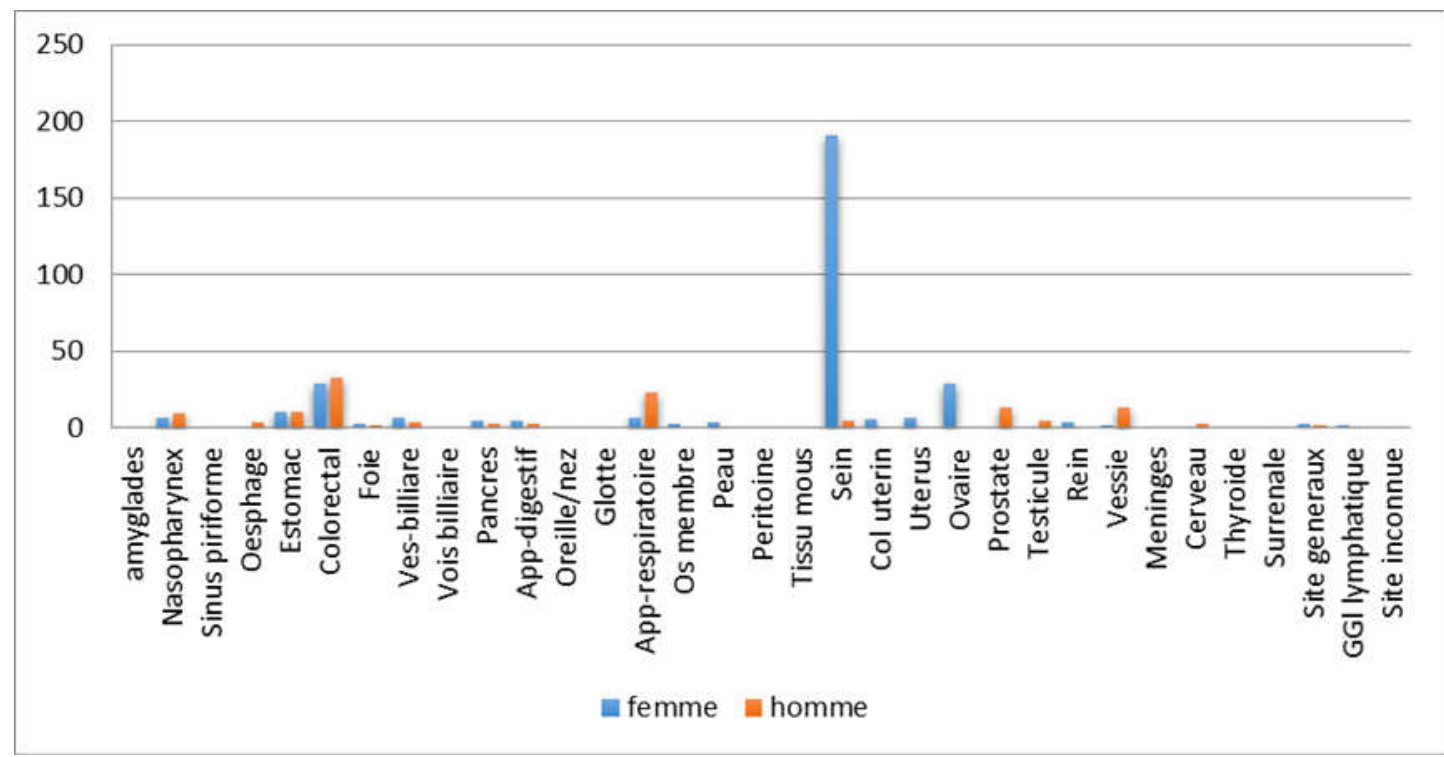

Fig.1. Incidence des différents types de cancers diagnostiqués à l'Hôpital MEB de Jijel en fonction du sexe

Dans un troisième temps, nous avons examiné la répartition des CCR en fonction d'antécédents personnels et familiaux. Le Tableau 1 résume les principaux antécédents et les états précancéreux qui prédisposaient au développement des CCR. Concernant les antécédents personnels et hormis la présence de huit cas présentant des polypes adénomateux, nous avons observé la présence de quatre cas de maladies inflammatoires, deux cas de CCR et un cas de cancer non digestif; les autres sujets ne présentaient pas d'état précancéreux. 


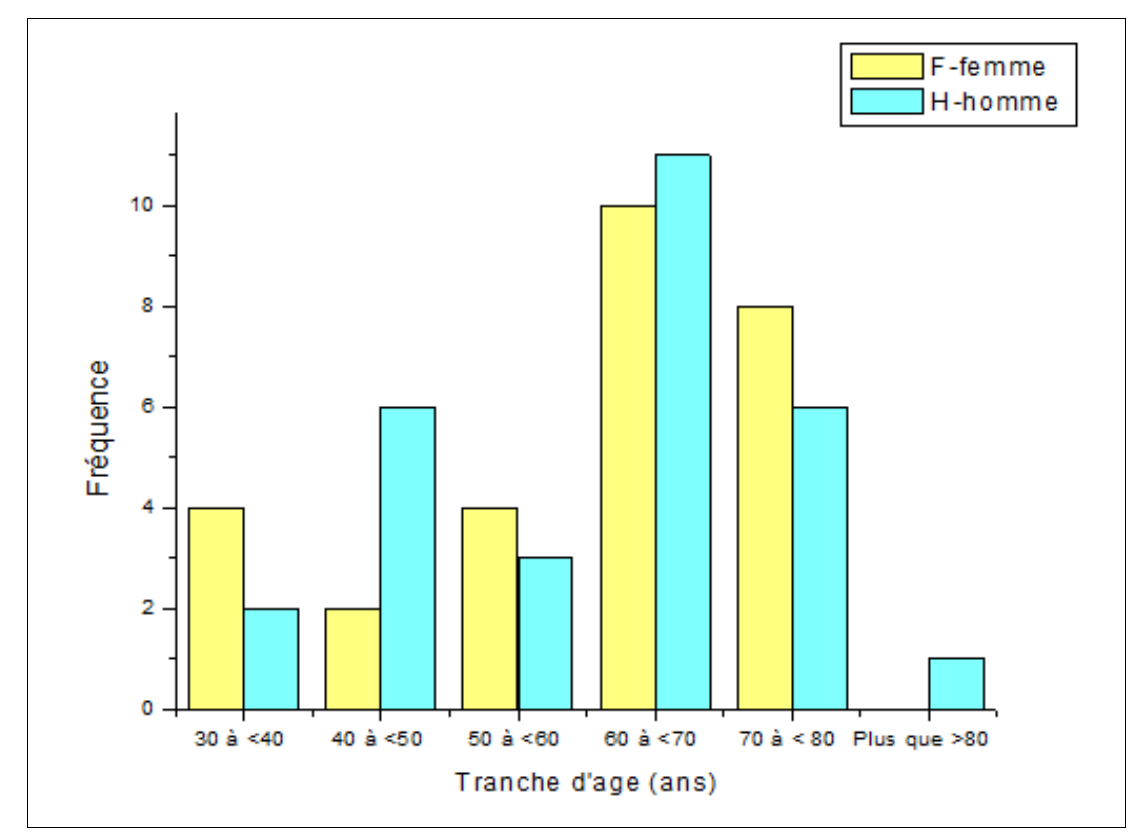

Fig.2. Répartition des CCR diagnostiqués à l'Hôpital MEB de Jijel en fonction du sexe et des tranches d'âges.

Tableau 1. Répartition des CCR en fonction des antécédents personnels et familiaux.

\begin{tabular}{lcc}
\hline Antécédents & Nombre de cas & Pourcentage (\%) \\
\hline Personnels & 08 & 12,90 \\
Polypes adénomateux & 04 & 06,45 \\
Maladie inflammatoire & 01 & 01,61 \\
Cancer non digestif & 10 & 16,13 \\
Diabète & 03 & 04,84 \\
Cholécystectomie & 00 & 00,00 \\
Acromégalie & 24 & 38,71 \\
Autre & & \\
Familiaux & 11 & 17,74 \\
Cancer digestif & 04 & 06,45 \\
Cancer non digestif & 01 & 01,61 \\
Polypose adénomateuse familiale & 00 & 00,00 \\
Syndrome de Lynch & 08 & 12,90 \\
Autre & \multicolumn{2}{l}{ L'étude a concerné 62 cas de CCR diagnostiqués à l'Hôpital MEB. } \\
\hline
\end{tabular}

S'agissant des principaux antécédents familiaux qui prédisposaient aux CCR, nous avons noté l'absence du syndrome de Lynch et la présence de 11 cas de cancer digestif $(12,90 \%)$ et de quatre cas de cancer non digestif (6,45\%). De plus, $98 \%$ des cas étaient de type sporadique, en 
accord avec des données précédemment rapportées qui stipulaient que 95\% des CCR étaient de type sporadique et 5\% étaient de type familial [1].

Enfin, nous avons analysé la répartition géographique des CCR à travers les différentes localités de la Wilaya. Le développement de l'épidémiologie descriptive permet de comparer les données d'incidence à travers le monde. Il y a une grande disparité géographique entre les pays industrialisés, caractérisés par le taux le plus élevé des CCR et les pays en voie de développement dont l'Algérie qui sont connus par un taux relativement faible de ce type de cancers $[3,10]$. Cependant, cette faible fréquence tendait à augmenter de manière constante en raison de l'occidentalisation brusque du mode de vie et des habitudes alimentaires des algériens. La Figure 03 représente la répartition géographique des CCR.

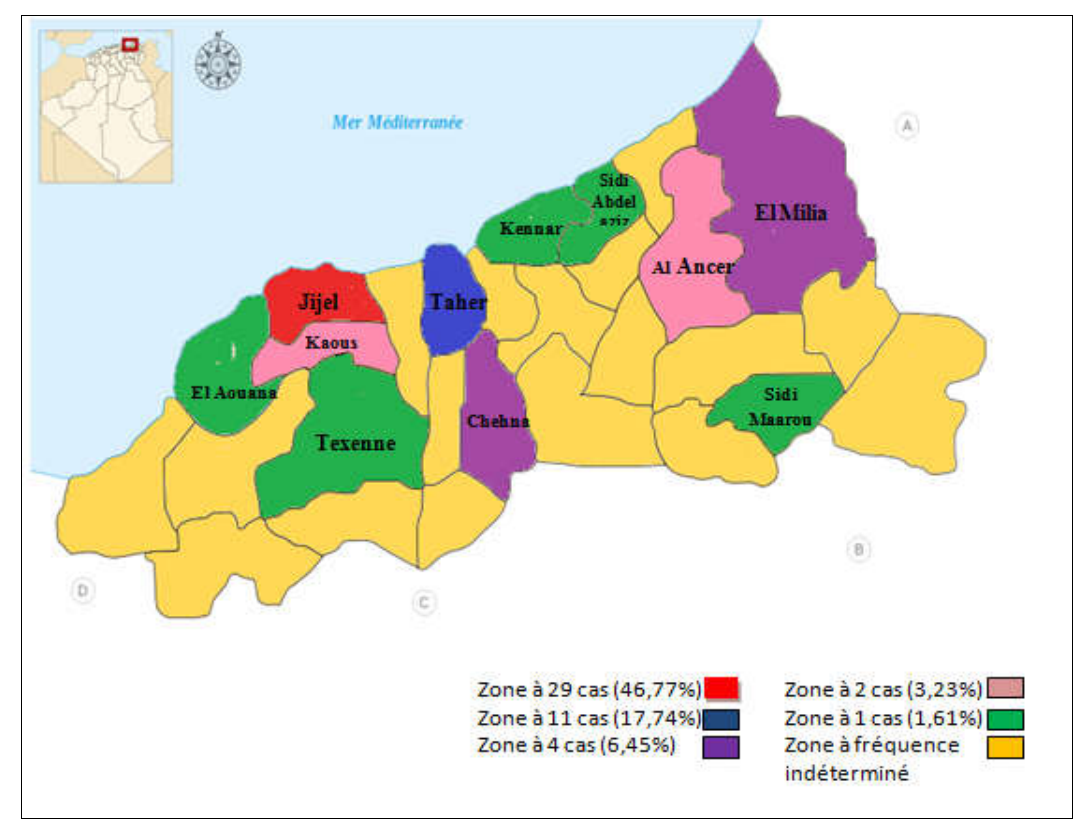

Fig.3. Répartition géographique des CCR diagnostiqués à l'Hôpital MEB de Jijel

Nous avons observé que la majorité des cas de CCR diagnostiqués $(46,77 \%)$ était localisée dans le Chef-lieu de la Wilaya, suivi par la région de Taher (17,74\%), une localité mitoyenne. Ces fréquences élevées pourraient être expliquées, du moins en partie, par un meilleur accès aux moyens de diagnostic des habitants de ces deux localités, la singularité de leurs habitudes alimentaires et de leur mode de vie. 


\subsection{Profils cliniques}

Tout d'abord, nous avons analysé la répartition des CCR en fonction de sept signes cliniques révélateurs (Douleurs abdominales, état général, rectorragie, syndromes rectaux, troubles du transit, anorexie et syndromes sub-occlusifs). Nos résultats concernant les profils cliniques sont résumés dans le Tableau 2. Nous avons observé la présence de douleurs abdominales et de l'altération de l'état général chez $77,42 \%$ et $25,81 \%$ des patients, respectivement.

Tableau 2. Répartition des CCR en fonction des signes cliniques.

\begin{tabular}{lcc}
\hline Paramètre & Nombre de cas & Pourcentage (\%) \\
\hline Signes révélateurs & 48 & \\
Douleurs abdominales & 26 & 77,42 \\
Rectorragie & 16 & 41,94 \\
Altération de l'état général & 12 & 25,81 \\
Syndrome rectal & 12 & 19,35 \\
Troubles du transite & 01 & 19,35 \\
Syndrome sub-ccolusif & 11 & 01,61 \\
Autre & & 17,74 \\
Autres signes & 22 & \\
Masse palpable & 17 & 35,48 \\
Ascite & 12 & 27,41 \\
Adénopathie & 11 & 19,35 \\
Hépathomégalie & 01 & 17,74 \\
Anorexie & L'étude a concerné 62 cas de CCR diagnostiqués à l'Hôpital MEB de Jijel. \\
\hline
\end{tabular}

Les douleurs abdominales sont les symptômes révélateurs les plus fréquents qui poussent généralement le malade à venir consulter le médecin. Nous avons aussi remarqué une perte de poids et d'appétit, un abaissement de la tension artérielle, ainsi que d'autres signes. Le syndrome rectal a été observé chez 19,35\% des cas. L'hémorragie digestive sous forme de rectorragie, un signe révélateur alarmant, a permis de révéler la présence de CCR dans 41,94\% des cas. En outre, nous avons observé la présence du syndrome sub-occlusif chez $1,61 \%$ des cas et du trouble du transit chez 19,35\%. Ces résultats sont concordants avec les travaux réalisés par Konaté et coll. [11]. Cependant, ils contrastent avec ceux obtenus par Sentissi et coll. qui proposaient la rectorragie comme étant le signe révélateur le plus fréquent 
(57\%) [9]. Néanmoins, il est très utile de constater que l'ensemble de ces paramètres cliniques témoignaient de la présence d'un état avancé de la maladie chez la majorité des patients.

Contrairement aux pays occidentaux dans lesquels la proportion des CCR diagnostiqués au cours de la phase asymptomatique (stade précoce où le pronostic est meilleur) est estimée à 27\%, nous avons observé que le diagnostic des CCR dans notre Willaya était à un stade avancé de la pathologie. Ce constat peut être expliqué par l'absence de programmes de dépistage précoce des populations à risque élevé de développer des CCR. D’une façon générale, nous avons constaté une absence totale d'une politique nationale de dépistage de masse, ce qui expliquerait l'augmentation constante des taux de morbidité et de mortalité par ces pathologies. Enfin, nous avons remarqué la présence d'autres signes cliniques de type physique tels que l'épanchement d'un liquide séreux dans la cavité péritonéale (ascite), la présence d'une masse palpable, les adénopathies et les hépatomégalies qui révèlent des stades avancés de CCR (Tableau 2).

\subsection{Bilans des examens paracliniques}

L'examen endoscopique a été pratiqué sur la totalité des patients, nous avons constaté l'usage de la coloscopie $(79,03 \%)$ et la recto-sigmoïdoscopie $(43,55 \%)$ (Tableau 3), ce qui est tout à fait compatible avec les données rapportées par Sentissi et coll. [9]. En général, les patients ont été soumis à la coloscopie, un examen capable d'explorer la totalité de la partie colorectale et de fournir des biopsies, alors que la recto-sigmoïdoscopie et la rectoscopie étaient spécifiques de la région recto-sigmoïdienne seulement. En outre, des examens complémentaires (Scanner, TDM thoraco-abdomino-pelvien) ont été pratiqués sur l'ensemble des patients afin de rechercher les signes pulmonaires et hépato-abdomino-pelviens (Tableau 3).

Les examens endoscopiques ont permis de déterminer les sièges de la tumeur (Tableau 4). En effet, nous avons constaté que la jonction recto-sigmoïdienne était le siège le plus touché (40,32\%), suivi par la localisation colique $(34,83 \%)$ et le rectum $(24,19 \%)$, ce qui est concordant avec les résultats de Darré et coll. [12]. Aussi, nous avons remarqué que le nombre de patients ayant un cancer du rectum était égale au nombre de patients qui ont subi une rectoscopie avec $(\mathrm{p}=0,05)$. Un résultat similaire a également été obtenu lors de l'emploi de la 
recto-sigmoïdoscopie pour détecter le cancer recto-sigmoïdien $(\mathrm{p}=0,0005)$, ce qui confirmait la spécificité de ces deux examens à la région recto-sigmoïdienne.

Tableau 3. Répartition des CCR en fonction des données paracliniques

\begin{tabular}{lcc}
\hline Paramètre & Nombre de cas & Pourcentage (\%) \\
\hline Examens endoscopiques & 49 & 79,03 \\
Coloscopie & 27 & 43,55 \\
Recto-sigmoidoscopie & 15 & 24,19 \\
Rectoscopie & 04 & 06,45 \\
Fibroscopie & & \\
Examens complémentaires & 62 & 100,0 \\
Scanner abdomino-pelvien & 62 & 100,0 \\
TDM-TAP & 61 & 98,39 \\
Scanner thoracique & 09 & 14,52 \\
IRM & & \\
Nombre de lésions & 48 & 77,42 \\
Néant & 06 & 09,67 \\
Multible & 03 & 04,83 \\
Unique & 05 & 08,06 \\
Indéterminé & & \\
\hline L'étude a concerné 62 cas de CCR diagnostiqués à l'Hôpital MEB de Jijel. TDM-TAP : TDM \\
thoraco-abdomino pelvien.
\end{tabular}

Tableau 4. Répartition des CCR en fonction du siège de la tumeur et du sexe.

\begin{tabular}{lccc}
\hline Localisation & Nombre de Femmes & Nombre d'Hommes & Total \\
\hline Recto-sigmoïdienne & 17 & 08 & $\mathbf{2 5}$ \\
Colon & 08 & 14 & $\mathbf{2 2}$ \\
Rectum & 04 & 11 & $\mathbf{1 5}$ \\
Total & $\mathbf{2 9}$ & $\mathbf{3 3}$ & $\mathbf{6 2}$ \\
\hline
\end{tabular}

L'étude a concerné 62 cas de CCR diagnostiqués à l'Hôpital MEB de Jijel.

Par ailleurs, le nombre des coloscopies réalisées était largement supérieur au nombre des cancers du côlon, étant donné que la coloscopie n'est pas spécifique de la région colique. En effet, elle est capable d'explorer la totalité de la partie colorectale et de fournir des biopsies, ce qui permet son utilisation comme examen endoscopique de référence pour le pronostic et le diagnostic des CCR. Cependant, elle est inappropriée pour la détection des adénomes de moins de $1 \mathrm{~cm}$ qui nécessite d'autres examens tels que la chromo-endoscopie, une nouvelle 
technique endoscopique qui fait appel à des colorants de contraste [13].

Nous avons également noté que les hommes étaient les plus touchés par le cancer du côlon (14 cas) et du rectum (11cas), alors que les femmes étaient les plus touchées par le cancer recto-sigmoïdien (17 cas, $\mathrm{p}=0.003$ ) (Tableau 4). La différence des sièges de la localisation entre les femmes et les hommes était significative.

Le nombre des lésions peut aider à déterminer la forme des CCR, on parle de polype sporadique (un à quelques éléments) ou de polypose (10 à 100 éléments ou plus). La plupart des polyposes sont héréditaires et associées à un risque élevé de cancer [14]. Nous avons noté la présence de six cas $(9,68 \%)$ ayant des lésions multiples réparties entre la partie colique (2 cas), la partie recto-sigmoïdienne ( 1 cas) et le rectum $(3$ cas $)$ et trois autres cas $(4,84 \%)$ présentant une lésion unique localisée dans la jonction recto-sigmoïdienne (Tableau 3).

Par ailleurs, l'endoscopie permet de déterminer l'aspect macroscopique des CCR. Ainsi, l'aspect infiltrant était majoritaire (79,03\%), suivi par l'aspect bourgeonnant (74,19\%) et puis ulcérant (70,97\%) (Tableau 5). Ces résultats contrastent avec les conclusions de Konaté et coll. qui ont démontré que la forme dominante était la forme ulcéro-bourgeonnante [11].

Enfin, le bilan d'extension permet de détecter les différentes localisations secondaires. Ainsi, nous avons observé la présence d'adénopathies chez 62,90\% des patients et de métastases ganglionnaires $(50 \%)$ et hépatiques $(30,65 \%)$ (Figure 4). Ceci contraste avec les résultats d'une étude française qui ont suggéré une proportion de 14,5\% seulement [15]. 
Tableau 5. Répartition des CCR en fonction des données anatomopathologiques

\begin{tabular}{lcc}
\hline Caractéristique & Nombre de cas & Pourcentage (\%) \\
\hline Aspects macroscopique & 49 & 79,03 \\
Infiltrant & 46 & 74,19 \\
Bourgeonnant & 44 & 70,97 \\
Ulcérant & 19 & 30,65 \\
Sténosant & 03 & 04,84 \\
Végétant & & \\
Architecture histologique & 44 & 70,97 \\
Indéterminé & 07 & 11,29 \\
Tubuleux papillaire & 04 & 06,45 \\
Tubuleux & 04 & 06,45 \\
Tubuleux villeux & 03 & 04,84 \\
Villeux & & \\
Degré de Différenciation & 40 & 64,52 \\
Différencié & 05 & 08,06 \\
Moyennement différencié & 04 & 06,45 \\
Indifférencié & 13 & 20,97 \\
Indéterminé &
\end{tabular}

\subsection{Profils anatomopathologique et histologique}

La répartition des $\mathrm{CCR}$ en fonction d'examens anatomopathologiques est une étape importante dans le protocole d'évaluation de l'extension de la maladie [16]. Ainsi, nous avons analysé les tissus tumoraux réséqués lors de l'endoscopie. Les données de ces examens ont permis de confirmer le diagnostic des CCR et révélaient les caractéristiques spécifiques des tumeurs (Tableau 05). Les adénocarcinomes ont représenté la variété histologique prédominante (98\%), ce qui est compatible avec les données rapportées par Konaté et coll. [11]. Presque la quasi-totalité des adénocarcinomes était de type Lieberkühnien et la proportion restante $(2 \%)$ représentait des sarcomes.

Le degré de différenciation cellulaire nous a permis de classer les tumeurs en adénocarcinomes bien différenciés $(64,52 \%)$, moyennement ou peu différenciés $(8,06 \%)$, indifférenciés $(6,45 \%)$ et indéterminés $(20,97 \%)$, ces proportions sont proches des valeurs rapportées par Darré et coll. [12]. L'examen de l'architecture des adénocarcinomes a révélé que $11,29 \%$ des cas étaient de type tubulo-papillaire (Tableau 05). 


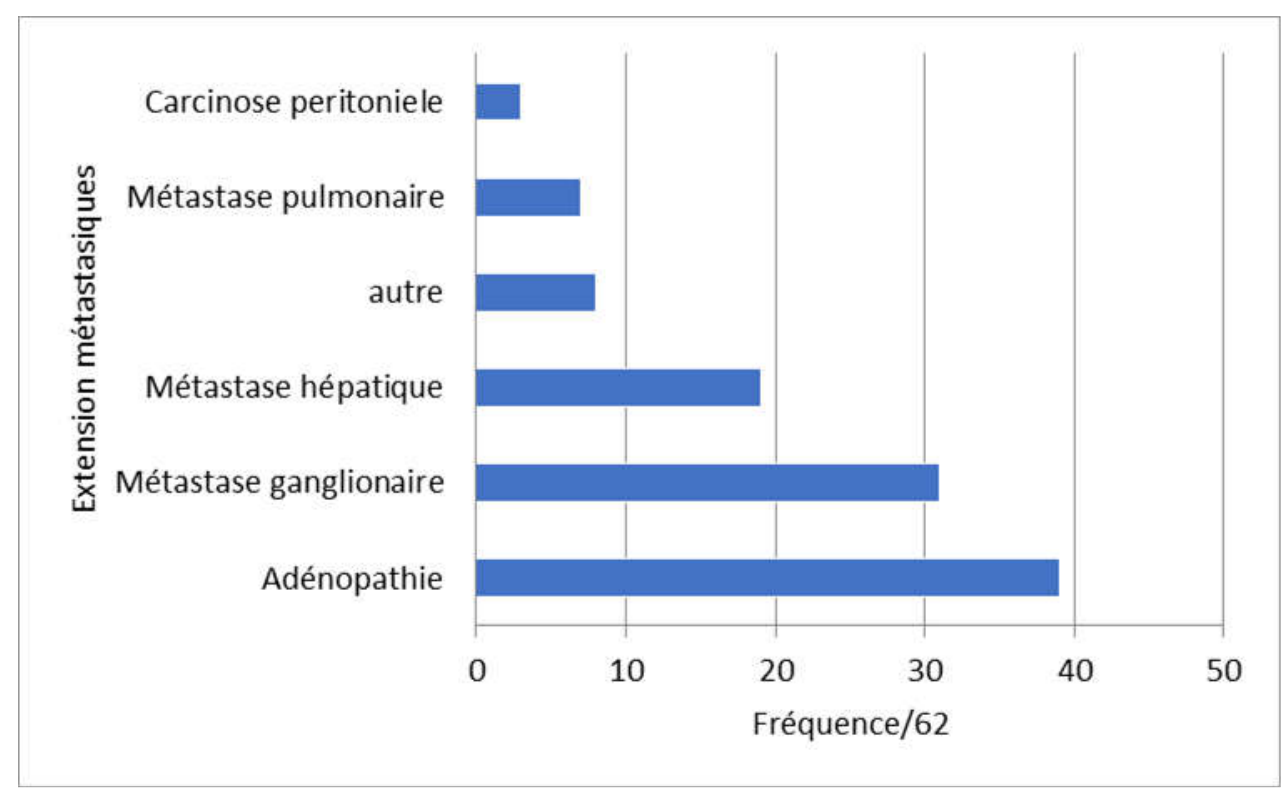

Fig.4. Répartition des CCR diagnostiqués à l'Hôpital MEB de Jijel en fonction des extensions métastasiques.

La classification TNM et UICC des CCR [17], a permis d'individualiser quatre stades de la maladie : I, II, III et IV (Tableau 06). L'évaluation du grade histo-pronostique TNM a permis de noter que la population étudiée présentait une prédominance du stade IIA $(24,19 \%)$. Au cours de ce stade, les cellules tumorales commençaient à envahir la musculeuse, sans présenter une métastase ni ganglionnaire, ni à distance [18]. Le stade IIIB a aussi partagé la même fréquence que celle du stade IIA, ce qui signifie que les ganglions lymphatiques étaient atteints par les cellules cancéreuses en envahissant la sous séreuse au passage.

Les tumeurs de stade I ont été localisées dans le tissu intra-muqueux et totalisaient $6,45 \%$ des cas, tandis que celles de stade IV en présentaient 3,23\%. Ce dernier est le plus critique, il définit les CCR qui se sont étendus aux organes voisins et qui ont pu métastaser. Aussi, nous avons remarqué que la fréquence des patients de stades précoces était très faible $(6,45 \%)$ comparativement à celle de stades avancés (72,58\%), sachant que 20,97\% étaient indéterminés, ceci peut être expliqué par l'absence d'une politique nationale de dépistage précoce. Cependant, nous avons noté une compatibilité entre nos résultats et ceux rapportés par une étude de cohorte européenne menée par Romaguera et coll. [19]. En effet, la 
fréquence des CCR précoces était faible, il semblerait qu'elle n'était pas liée à l'absence d'un dépistage précoce, mais plutôt liée à des facteurs de risques comme le régime alimentaire.

Tableau 6. Répartition des CCR en fonction de la classification TNM établie par l'OMS

\begin{tabular}{|c|c|c|c|}
\hline Classe & Nombre de cas & Pourcentage (\%) & Pourcentage cummulé (\%) \\
\hline Stade I & 04 & 06,45 & 06,45 \\
\hline Stade IIA & 15 & 24,19 & 30,64 \\
\hline Stade IIB & 01 & 01,61 & 32,25 \\
\hline Stade IIIA & 06 & 09,68 & 41,93 \\
\hline Stade IIIB & 15 & 24,19 & 66,12 \\
\hline Stade IIIC & 06 & 09,68 & 75,80 \\
\hline Stade IV & 02 & 03,23 & 79,03 \\
\hline Indéterminé & 01 & 20,97 & 100,0 \\
\hline Total & 62 & 100,0 & 100,0 \\
\hline
\end{tabular}

Les caractéristiques histologiques des tissus appropriés chez les patients atteints de CCR sont un moyen incontournable pour évaluer l'état d'avancement de la maladie. Ainsi, nous avons analysé les aspects histo-pathologiques de deux cas de CCR à titre d'exemples. Le premier était de sexe masculin, âgé de 63 ans, atteint d'un CCR sporadique de stade IIIA et présentait une métastase ganglionnaire. Le deuxième était de sexe féminin, âgé de 63 ans, atteint d'un CCR de stade IIIC, sans antécédents familiaux et présentait une mutation du gène K-RAS. Nous avons observé que le premier patient était atteint d'un adénocarcinome Lieberkühnien bien différencié, les glandes étaient de taille variable et tapissées par un épithélium pluristratifié sous forme tubulo-papillaire (Figure 5 : B et C). Les noyaux étaient monstrueux, vésiculeux, de taille augmentée et caractérisés par la présence d'une chromatine hétérogène et d'un stroma fibro-vasculaire grêle remmené par la nécrose. 

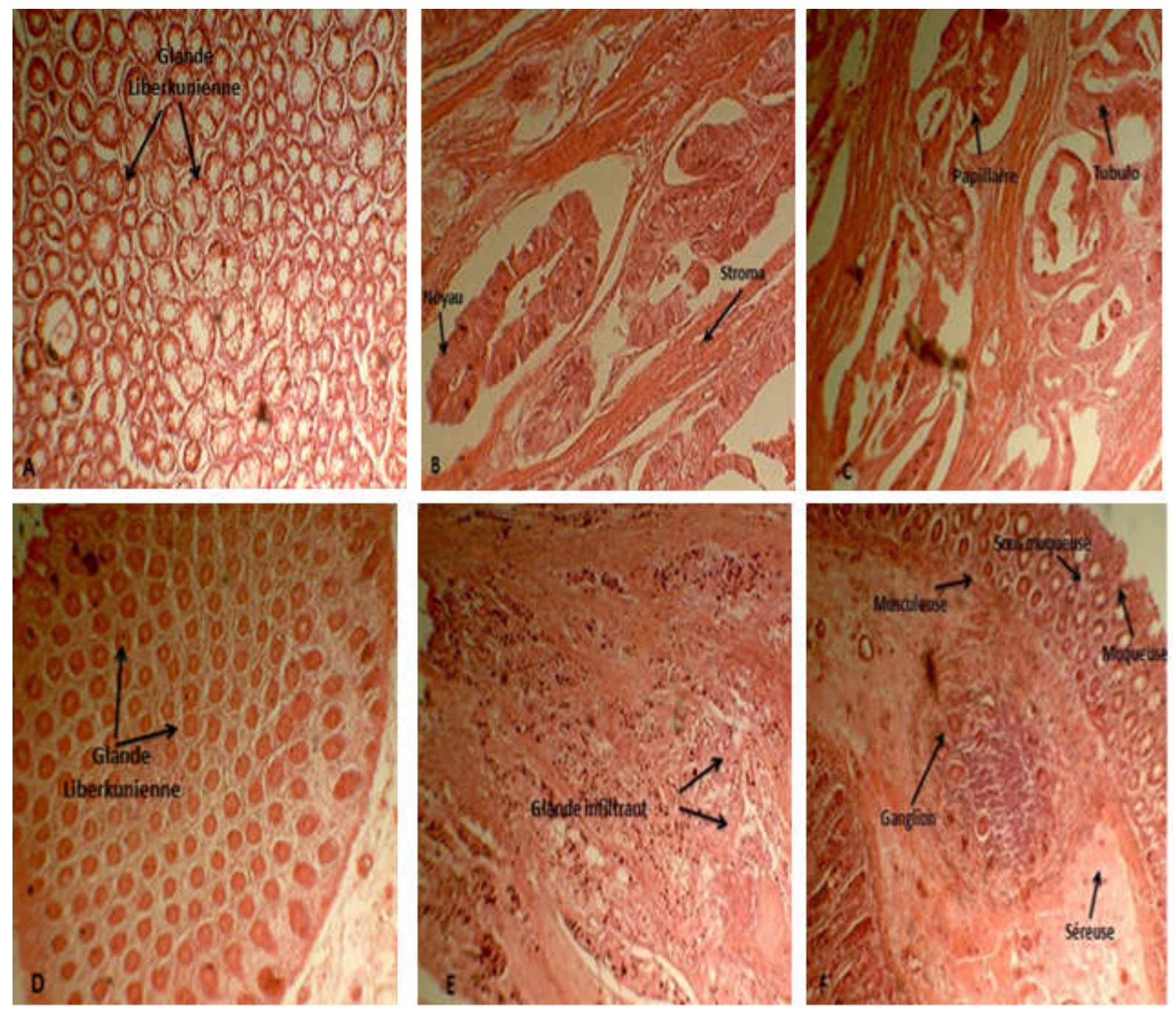

Fig.5. Analyse histologique par microscopie optique (G 4X10) de tissus cancéreux de deux cas de CCR. A, B et C) Patient atteint d'un CCR dont le gène K-Ras est normal ; A : tissu sain ; B $+\mathrm{C}$ : adénocarcinome Lieberkühnien bien différencié, d'architecture tubulo-papillaire avec noyau de taille augmenté et le stroma fibro-vasculaire grêle par nécrose. D, E et F) Patient atteint d'un CCR dont le gène K-RAS est muté ; D : tissu sain ; E+F : adénocarcinome Lieberkühnien moyennement différencié, caractérisé par des glandes de taille variable et la présence d'adénopathies infiltrant la sous-muqueuse.

Parallèlement, nous avons noté que le deuxième patient présentait un adénocarcinome Lieberkühnien moyennement différencié et caractérisé par une infiltration sous forme massive creusée par des cavités qui s'accompagnait par un aspect cribyforme (Figure 5 : E et F). Les glandes étaient de taille variable et caractérisées par des atypies cyto-nucléaires sévères, le tout accompagné d'adénopathies réactionnelles de la sous muqueuse. A noter que les glandes 
Lieberkühniènnes des tissus sains de chacun des deux patients étaient de taille régulière (Figure 5 : A et D).

La comparaison des degrés de différenciation entre les deux patients, nous a permis de constater que le patient porteur du gène K-RAS normal présentait un adénocarcinome bien différencié, alors que le patient porteur du gène K-RAS muté correspondait à un adénocarcinome moyennement différencié. Ceci signifie que la mutation du gène K-RAS semble ne pas avoir d'effet très marqué sur le degré de différenciation des tumeurs.

\subsection{Bilans des examens biologique et moléculaire}

Nous avons analysé la répartition des CCR en fonction des bilans biologiques, en évaluant les taux des marqueurs tumoraux et hépatiques, ainsi que ceux des globules blancs et rouges (Tableau 07). Nous avons observé un taux élevé de l'ACE chez 45,16\% des cas et une valeur importante de CA19-9 chez 25,29\% des cas. L'augmentation des taux de ces deux marqueurs est un indicateur de l'état d'avancement de la maladie [20].

Tableau 7. Répartition des CCR en fonction des bilans biologiques

\begin{tabular}{|c|c|c|}
\hline Paramètre & Nombre de cas & Pourcentage (\%) \\
\hline \multicolumn{3}{|c|}{ Marqueurs tumoraux (A) } \\
\hline $\mathrm{ACE}$ & 28 & 45,16 \\
\hline CA19-9 & 16 & 25,81 \\
\hline \multicolumn{3}{|c|}{ Bilans hépatiques (A) } \\
\hline ASAT & 09 & 14,52 \\
\hline ALAT & 08 & 12,90 \\
\hline PAL & 18 & 29,03 \\
\hline \multicolumn{3}{|c|}{ Bilans hépatiques } \\
\hline Globules rouges & 29 & 46,77 \\
\hline Globules blanc & 03 & 04,84 \\
\hline
\end{tabular}

La moitié des cas présentaient des métastases hépatiques et ganglionnaires caractérisées par une prédominance colique de la localisation primaire, ce qui est tout à fait en accord avec les travaux de Vukobrat et coll. [21]. 
La corrélation entre les métastases hépatiques et les marqueurs hépatiques a été démontrée par l'existence d'une relation hautement significative $(\mathrm{p}=0.0001)$ entre les patients ayant des taux élevés de PAL (12 cas) et les métastases hépatiques (19 cas). Ces résultats sont compatibles avec les conclusions d'un travail réalisé par Saif et coll. [22]. Par contre, la relation entre métastases hépatiques et les transaminases (Alanine aminotransférase ou ALAT, aspartate aminotransférase ou ASAT) n'a pas été significative $(p=0.25)$. S'agissant des bilans hématologiques, nous avons noté un taux faible des globules rouges chez $46,77 \%$ des cas et qui pourrait être due à la présence d'hémorragies coliques ou rectales $(\mathrm{p}=0.4)$ (Tableau 07).

Enfin, l'analyse des profils moléculaires de neuf patients a révélé que les deux tiers des cas présentaient des mutations du gène K-RAS réparties entre l'exon 2 (5 cas) et l'exon 4 (1 cas), ces résultats sont conformes à des travaux récemment rapportés [23,24] (Données non présentées). Le tiers restant (3 cas) était des hommes possédant le gène K-RAS sauvage. Ces données ont permis de confirmer l'existence d'une corrélation entre la mutation de l'exon 2 du gène K-RAS et le sexe des patients, en synergie avec des résultats de Li et coll. [25].

La tumeur était localisée dans la jonction recto-sigmoïdien chez quatre patients dont le gène K-RAS était muté. Les CCR étaient présents dans des stades tardifs selon la classification TNM et UICC ; nous avons trouvé un seul cas de stade IIA, trois cas de stade IIIB et deux cas de stade IIIC. Nous avons également observé que plus de la moitié des patients (4 cas) ayant le gène K-RAS muté présentaient des métastases ganglionnaires et des adénopathies, alors que deux cas avaient des métastases hépatiques. La mutation du codon 12, et non pas celle du codon 13 de l'exon 2 du gène K-RAS est associée aux métastases ganglionnaires et les stades avancés de CCR, ce qui conforte nos résultats [25,26].

\subsection{Profils thérapeutiques}

Nous avons cherché à établir des corrélations potentielles entre la répartition des CCR et les différentes thérapeutiques employées (Chirurgie, radiothérapie, chimiothérapie et immunothérapie). Nous avons constaté que $66,12 \%$ des patients (41 cas) ont bénéficié d'une chirurgie curative (Figure $6:$ A). Le seul patient qui a bénéficié d'une chirurgie palliative avait un antécédent familial de type PAF, il a subi une résection totale du colon pour éviter le 
développement inéluctable de la maladie [27]. Sept patients ont fait l'objet d'une radiothérapie comme moyen de guérison. S'agissant de la chimiothérapie, 58,06\% des patients ont été la cible d'une chimiothérapie adjuvante et 19,35\% d'une chimiothérapie néo-adjuvante, et le protocole le plus appliqué était le XELOX+BEVA (Figure $6: \mathrm{B}$ ). Ce protocole est une association entre la chimiothérapie classique (Oxaliplatine associé au Capecitabine : XELOX) [28] et la thérapie ciblée (Bevacizumab) ${ }^{+}$. L'addition des antimitotiques récents tels que 5-Fluorouracile (5-FU), leucovorin, irinotecan (FOLFIRI) et Oxaliplatin (FOLFOX) en fortes concentrations a permis de diminuer la dissémination des cellules tumorales par la voie portale et péritonéale, permettant ainsi une diminution des récidives hépatiques et péritonéales [29,30].

Le protocole XELOX+BEVA était aussi le plus appliqué chez les patients ayant le gène K-RAS muté. L'efficacité du traitement au Cétuximab et Panitumab varie selon la présence ou l'absence des mutations du gène K-RAS. Plusieurs études ont montré que ces médicaments n'agissaient qu'en absence de mutations de ce gène [31,32], ce qui explique l'utilisation intense de Bevacizumab chez ces patients porteurs du gène K-RAS muté. De nombreuses études combinant la chimiothérapie avec Bevacizumab ont montré une efficacité augmentée par rapport à la chimiothérapie seule, ce médicament de la thérapie ciblée anti-VEGF est utilisé pour le traitement des CCR métastatiques. C'est un anticorps monoclonal qui cible le facteur de croissance endothélial vasculaire, une protéine impliquée qui joue un rôle important dans l'angiogenèse colorectale [33]. Il améliore également l'action des agents chimio-thérapeutiques en favorisant l'acheminement de ces médicaments directement vers la tumeur. Il est généralement administré en association avec d'autres médicaments, comme le 5-FU, l'oxaliplatine, l'irinotécan et la capécitabine [32]. Les deux autres médicaments Cetuximab et le Panitumab sont des anticorps monoclonaux chimériques, utilisés en thérapie ciblée [34]. Ces anticorps ciblent les récepteurs du facteur de croissance épidermique (EGFR), exprimés sur la surface des cellules cancéreuses dans $80 \%$ des cas de CCR [35, 32]. 


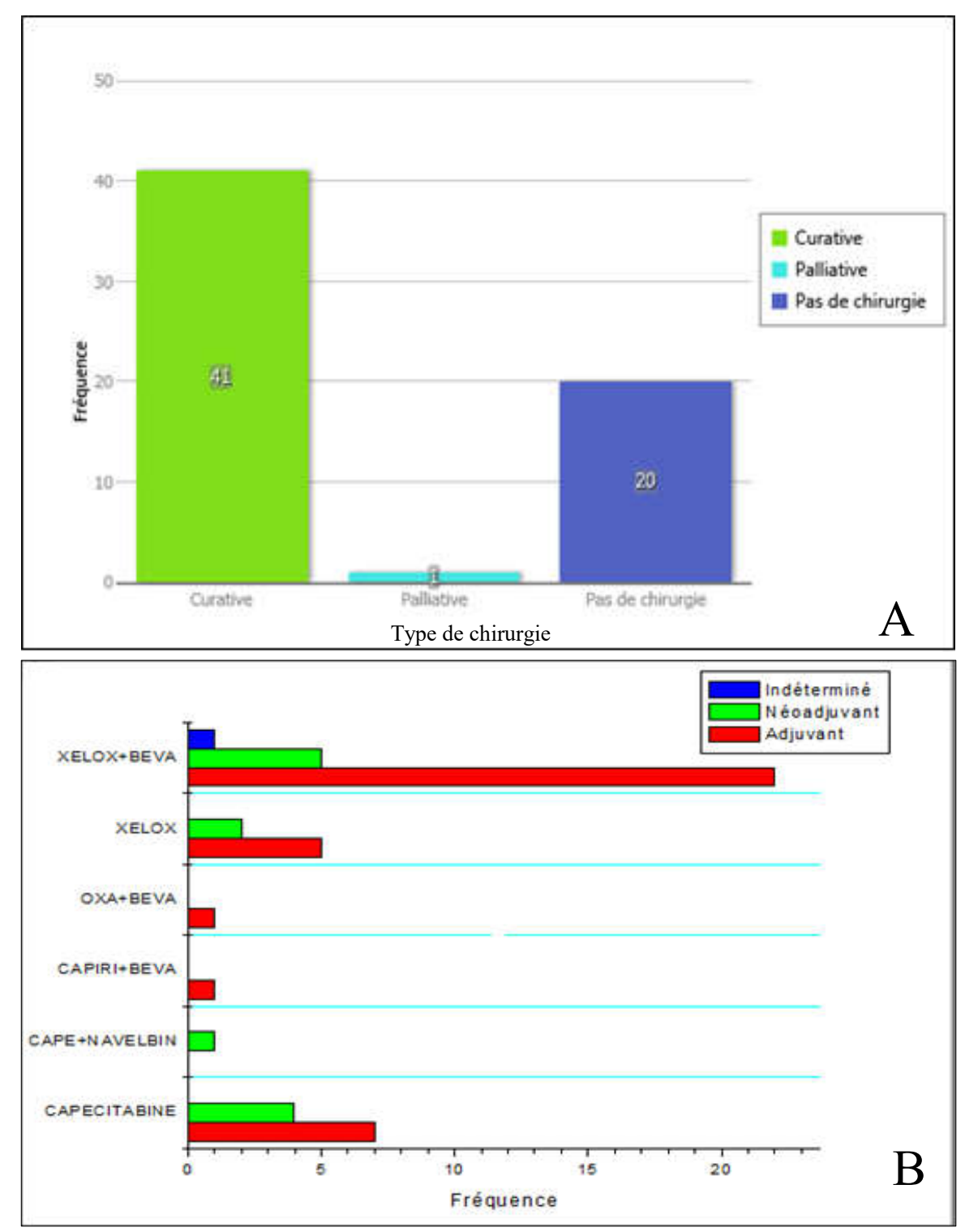

Fig.6. Répartition des CCR en fonction du type de Thérapie. A : chirurgie ; chimiothérapie.

L'étude a concerné 62 cas de CCR diagnostiqués à l'Hôpital MEB de Jijel

\section{CONCLUSION}

Notre étude rétrospective et descriptive a reposé sur une analyse des aspects épidémiologique, clinique, paraclinique et biologique de 62 patients atteints de CCR et qui permettrait désormais de fournir des données fiables et pertinentes sur cette maladie. En dépit du fait que le nombre de patients étudiés était relativement limité, nos résultats ont été souvent compatibles avec les données disponibles. Nous avons démontré que les CCR occupaient la $2^{\text {ème }}$ place par rapport à l'ensemble des cancers et la $1^{\text {ère }}$ place parmi les cancers digestifs avec 
une prédominance de la localisation colique. La tranche d'âges la plus touchée était 60-70 ans, avec une prédominance masculine et un âge moyen précoce comparativement aux pays occidentaux dont les populations sont vieillissantes. Ces résultats sont aussi concordants avec d'autres études algériennes et maghrébines auxquelles nous avons pu accéder. De plus, nous avons constaté que le plus grand nombre des patients résidaient au Chef-lieu de la Willaya, suivi de la région de Taher. Cette observation différentielle s'expliquerait par la sédentarité des populations concernées, l'enclavement de ces deux régions de la willaya ainsi que par la singularité du mode de vie et des habitudes alimentaires adoptés par les habitants de ces deux localités.

Sur le plan clinique, les patients n'ont bénéficié d'une coloscopie qu'après l'apparition des signes révélateurs de la présence de la tumeur, ce qui confirme l'absence d'un programme de dépistage précoce. Le traitement de référence des CCR a été la chirurgie, elle permet d'éliminer la tumeur seulement si cette pathologie est dépistée à des stades suffisamment précoces où la tumeur est accessible à l'exérèse. Si la tumeur est non opérable, en cas des métastases, la chimiothérapie peut prendre place pour améliorer le traitement. Le 5-FU a été le médicament dominant dans l'arsenal de la chimiothérapie, il était employé seul ou combiné à d'autres drogues. Malheureusement, cette approche thérapeutique n'est pas sélective et inefficace, ce qui nécessite le recours à l'immunothérapie utilisant des anticorps monoclonaux comme le bevacizumab.

Bien que des progrès diagnostiques et thérapeutiques importants aient été réalisés, on ne guérit actuellement qu'un CCR sur deux. C'est la raison pour laquelle, à l'heure actuelle, le meilleur moyen de lutter contre cette maladie reste la prévention. On peut recommander de ne pas fumer, de ne pas boire de l'alcool, de manger beaucoup de légumes et fruits riches en fibres, d'éviter les excès alimentaires et d'avoir une activité physique dans le cadre du travail et des loisirs. Il est probable que ces mesures diminuent d'au moins un tiers le risque des CCR. Enfin, l'état actuel des connaissances nous permet de préconiser une politique de prévention secondaire et de dépistage en vue de réduire la mortalité et la morbidité des CCR. 


\section{ACKNOWLEDGEMENTS}

L'ensemble des auteurs tient à souligner l'aide et l'appui apportés par le Dr SAHALI Ilham de l'Unité d'Oncologie (Hôpital de Jijel) et le Dr BELGHAYET (Laboratoire d'Hygiène de Jijel).

Conflit d'intérêt : Les auteurs déclarent ne pas avoir de conflit d'intérêt.

\section{REFERENCES}

[1] Bogaert J, Prenen H. Molecular genetics of colorectal cancer. Ann Gastroenterol 2014; 27: 9-14.

[2] Gustavsson B, Carlsson G, Machover D, Petrelli N, Roth A, Schmoll HJ, et al. A review of the Evolution of Systemic Chemotherapy in the Management of Colorectal Cancer. Clin Colorectal Cancer 2015;14: 1-10.

[3] Aleksandrova K, Pischon T, Jenab M. Combined impact of healthy lifestyle factors on colorectal cancer: a large European cohort study. BMC Medicine 2014; 12: 168.

[4]. Abderrahmane R, Louhibi L, Moghtit FZ, Boubekeur A, Benseddik K, Boudjema A, et al. TP53 Arg 72Pro and MDM2 SNP309 Polymorphisms and Colorectal Cancer Risk: A West Algerian Population Study. Pathol Oncol Res 2015; 21: 629-35.

[5] Hamdi Cherif M, Serraino D, Mahnane A, Laouamri S, Zaidi Z, Boukharouba H, et al. Time trends of cancer incidence in Setif, Algeria, 1986-2010: an observational study. BMC Cancer 2014; 14: 637.

[6] Wang H, Gies N, Wong C, Sadowski D, Moysey B, Fedorak RN, et al. Patients undergoing colorectal cancer screening underestimate their cancer risk and delay presentation for screening. Can J Gastroenterol 2012; 26: 419-23.

[7] El Housse H, Ajbara W, Amsaguine S, El Amrani N, Drissi H, Ahallat M, et al. Profils épidémiologique et anatomoclinique d'une population marocaine atteinte de cancer colorectal. J Afr Cancer 2015; 7 : 95-9.

[8] Meddah D, Meddah B, Tir TA, Ghalek M, Sahraoui T. Étude épidémiologique du cancer du côlon chez des patients de l'Ouest. J Afr Cancer 2009; 1 : 31-5.

[9] Sentissi SL, Galab M, Amrani L, Chaoui Z., Kabbaj N., Guedira M, et al. Epidemiological 
characteristics profile of the colorectal cancer: a multicentric study. Arab J Gastroenterol 2009; 2: AB11.

[10] Esteban-Jurado C, Garre P, Vila M, Lozano JJ, Pristoupilova A, Beltrán S, et al. New genes emerging for colorectal cancer predisposition. World J Gastroenterol 2014; 20: 1961-71.

[11] Konaté I, Sridi A, Ba PA, Cissé M, Gaye M, Ka I, et al. Etude descriptive des cancers colorectaux à Clinique chirurgicale du CHU Aristide Le Dantec de Dakar. J Afr Cancer 2012; 4 : 233-7.

[12] Darré T, Amégbor K, Bagny A, Bagny A, Bouglouga O, Lawson AL, et al. Profil histo-épidémiologique des cancers colorectaux au Togo. J Afr Hépatol Gastroentérol 2014; 8 : 226-9.

[13] Gabbani T, Manetti N, Bonanomi AG, Annese AL, Annese V. New endoscopic imaging techniques in surveillance of inflammatory bowel disease. World J Gastrointest Endosc 2015; 7: 230-6.

[14] Benamouzig R, Chaussade S, Olschwang S. Les polyposes coliques : Attitudes pratiques. Gastroenterol Clin Biol 2005; 29 : 1035-9.

[15] Manfredi S, Lepage C, Hatem C, Coatmeur O, Faivre J, Bouvier AM. Epidemiology and Management of Liver Metastases From Colorectal Cancer. Ann Surg 201; 244: 254-9.

[16] Marzouk O, Schofield J. Review of Histopathological and Molecular Prognostic Features in Colorectal Cancer. Cancers 2011; 3: 2767-810.

[17] Bosman FT. Les biomarqueurs prédictifs dans le cancer colorectal. Rev Med Suisse 2009; $211: 1513-18$.

[18] Sobin LH, Hermanek P, Hutter RV. TNM Classification of Malignant Tumors. A Comparison Between the New (1987) and the Old Editions. Cancer 1988; 61: 2310-4.

[19] Romaguera D, Ward H, Wark PA, Vergnaud AC, Peeters PH, van Gils CH, et al. Pre-diagnostic concordance with the WCRF/AICR guidelines and survival in European colorectal cancer patients: a cohort study. BMC Medicine 2015; 13: 107.

[20] Strambu V, Garofil D, Pop F, Radu P, Bratucu M, Popa F. Translating clinical research of Molecular Biology into a personalized, multidisciplinary approach of colorectal cancer 
patients. J Med Life 2014; 7: 17-26.

[21] Vukobrat-Bijedic Z, Husic-Selimovic A, Sofic A, Bijedic N, Bjelogrlic I, Gogov B, et al. Cancer Antigens (CEA and CA 19-9) as Markers of Advanced Stage of Colorectal. Med Arch; 201367: 397-401.

[22] Saif MW, Alexander D, Wicox CM (2005) Serum Alkaline Phosphatase Level as a Prognostic Tool in Colorectal Cancer: A Study of 105 patients. J Appl Res; 20135: 88-95.

[23] Knickelbein K, Zhang L. Mutant K-RAS as a critical determinant of the therapeutic response of colorectal cancer. Genes Dis 2015; 2: 4-12.

[24] Bando H, Yoshino T, Shinozaki E, Nishina T, Yamazaki K, Yamaguchi K, et al. Simultaneous identification of 36 mutations in KRAS codons 61and 146, BRAF, NRAS, and PIK3CA in a single reaction by multiplex assay kit. BMC Cancer 2013; 13: 405.

[25] Li W, Qiu T, Zhi W, Shi S, Zou S, Ling Y, et al. Colorectal carcinomas with KRAS codon 12 mutation are associated with more advanced tumor stages. BMC Cancer 2015; 15: 340.

[26] Chen J, Guo F, Shi X, Zhang L, Zhang A, Jin H, et al. BRAF V600E mutation and K-RAS codon 13 mutations predict poor survival in Chinese colorectal cancer patients. BMC Cancer 2014; 14: 802.

[27] Caspari R, Lamberti CH. Impact de la génétique moléculaire sur le dépistage du cancer colorectal héréditaire nonpolypoïde. Acta Endoscopica 2007; 37 : 165-79.

[28] Kawakami K, Nakamoto E, Yokokawa T, Sugita K, Mae Y, Hagino A, et al. Patients' self-reported adherence to capecitabine on XELOX treatment in metastatic colorectal cancer: findings from a retrospective cohort analysis. Patient Prefer Adherence 2015; 9: 561-7.

[29] Srinivas US, Dyczkowski J, Beißbarth T, Gaedcke J, Mansour WY, Borgmann K, et al. 5-Fluorouracil sensitizes colorectal tumor cells towards double stranded DNA breaks by interfering with homologous recombination repair. Oncotarget 2015; 6: 12574-86.

[30] Assenat E, Duffour J, Marc Ychou M. Modalités d'administration de l'irinotecan dans le traitement du cancer colorectal métastatique. Bull Cancer 2006; 93 : 507-15.

[31] Chang YY, Lin J K, Lin TC, Chen WS, Jeng KJ, Yang SH, et al. Impact of K-RAS mutation on outcome of patients with metastatic colorectal cancer. Hepatogastoonterology; 201461: 1946-53. 
[32] Heinemann V, Stintzing S, Kirchner T, Boeck S, Jung A. Clinical relevance of EGFRand KRAS-status in colorectal cancer patients treated with monoclonal antibodies directed against the EGFR. Cancer Treat Rev 2009; 35: 262-71.

[33] Pino MS, Chung DC. The chromosomal instability pathway in colon cancer. Gastroenterology 2014; 138: 2059-72.

[34] Feng QY, Wei Y, Chen JW, Chang W J, Ye LC, Zhu DX, et al (2014) Anti-EGFR and anti-VEGF agents: Important targeted therapies of colorectal liver metastases. World J Gastroenterol 20: 4263-75.

[35] Chaiadini E, Scapi E, Passardi A, Calistri D, Valgiusti M, Saragoni L, et al. EGFR methylation and outcome of patients with advanced colorectal cancer treated with cetuximab. Oncol Lett 2015; 9: 1432-8.

\section{How to cite this article:}

Abbes A, Rechreche H, Brinet R, Boulkhiout S, Souilah N, Benali M.Retrospective study of epidemiological, clinicopathological and biological profils of 62 colorectal cancers cases in jijel provence (algeria). J. Fundam. Appl. Sci., 2018, 10(1), 59-82. 